\title{
Influence of Transcranial Direct Current Stimulation on Lower Limb Muscle Activation and Balance Ability in Soccer Player
}

\author{
Dae Jung Yang, Seung Kyu Park, Yo Han Uhm \\ Department of Physical Therapy, Sehan University, Yeongam, Korea
}

Purpose: This study is to investigate influence of tDCS on lower limb muscle activity and balance ability in soccer player.

Methods: Sessions were conducted with 15 subjects in tDCS group and 15 in action observation training group for 20 minutes, 5 sessions a week, for 8 weeks. All soccer players underwent 30 minutes of plyometric training before main exercise. To evaluate lower limb muscle activation, rectus femoris and biceps femoris were taken measure using surface electromyogram system and to evaluate balance ability, surface area, whole path length, limited of stability were measured using biorescue.

Results: Regarding balance shown in surface area, whole path length, limited of stability and muscle activation in rectus femoris and biceps femoris, tDCS group showed more significant change than action bservation training group.

Conclusion: Therefore, intervention using tDCS is more effective in improving lower limb muscle activation and balance ability than action observation training.

Keywords: tDCS, Muscle activity, Balance ability

\section{INTRODUCTION}

In soccer, amongst many sports activities, various techniques and explosive actions transpire such as jumping, kicking, tackling, turning, sprinting, pace shifting, etc. during a game. ${ }^{1}$ Soccer, unlike other sports, requires repetitive and continuous execution of various techniques. ${ }^{2}$ Soccer demands absolute physical traits of endurance to move continuously for long period of time and strength, speed, and quickness for explosive strength in a moment. Also, agility and balance for faster direction change are necessary physical traits as well. $^{3}$

Physical traits dominate in soccer, and among 6 traits (strength, quickness, agility, flexibility, balance, and endurance), strength has powerful influence in many actions. Leg strength especially is one of the crucial components in athletes and allows jump and sprint with explosive leg strength. For such skill, many athletes are improving their physical competence with strength training and complex exercise. ${ }^{4}$ As all movements that take place during the game are

Received Nov 13, 2018 Revised Dec 26, 2018

Accepted Dec 26, 2018

Corresponding author Yo Han Uhm

E-mail uhmyo112@naver.com based on muscular contraction, physical traits like muscular strength, endurance, and power are essential factors in determining athlete's performance. ${ }^{5}$ Goal of soccer is to put ball into the opponent's goal post. While goals are made with head, torso, and feet, but foot work is predominant in playing the game of soccer and functions of a soccer player's leg are to adjust and balance body and performing skills like shoot, pass, and dribble flawlessly. ${ }^{6}$ There are many occasions where one must cut through defense with explosive speed and for stronger shooting and higher jump heading, stronger leg strength is demanded. ${ }^{7}$ As leg muscle is employed in jump, kick, tackle, turn, speeding, and turn, enhancement in its strength is relevant to improvement of such skills. ${ }^{8}$ Foundation for explosive movement lies in leg muscular strength and leg muscular power has much impact on game performance.

Balance can be classified into static balance and dynamic balance. Static balance is to maintain desired posture by placing gravitational center in base of support while body is static, whereas dynamic balance is to maintain desired posture by placing gravitational center

Copylight (C2018 The Korea Society of Physical Therapy

This is an Open Access article distribute under the terms of the Creative Commons Attribution Non-commercial License (Http:// creativecommons.org/license/by-nc/4.o.) which permits unrestricted non-commercial use, distribution, and reproduction in any medium, provided the original work is properly cited. 
in base of support while body is moving. Balance is the capacity to maintain posture by modulating locational relation between each body part according to gravitational or load change. ${ }^{10}$ Balance is crucial for better performance in sports such as soccer, basketball, and gymnastics, and especially, soccer demands one-foot posture to perform technical movements like shooting or pass and requires stability of supporting leg for precise execution. ${ }^{11}$ Balance helps with precise execution of various, technical movements including shooting, pass, and kick, and reduces risk of ankle and knee injury, improving game performance. ${ }^{12,13}$

The most typical training program for soccer players has incorporated programs diverse in training technique and time including weight training that focuses on enhancing muscular strength, overall endurance training with consistent interval and circuit training, and complex training comprising of both trainings. ${ }^{14} \mathrm{tDCS}$ is one of the most representative noninvasive equipment that can directly stimulate the brain and change excitability of brain cell, it can expedite or suppress the excitability to find out neuro-physiological function in each area of the brain and is being used in many different areas for recovery of performance. ${ }^{15,16} \mathrm{tDCS}$ that alternates excitatory of focal brain neuron by discharging electrical current onto scalp is convenient and induces and maintains selective functional alternation in brain cortex, of which application is being used in various fields of research. ${ }^{17}$ tDCS can alter brain function in its voluntary neural activity with brain neural polarization which employs direct current stimulation technology. ${ }^{18}$ Other non-invasive stimulation systems, TMS and rTMS, only affects relatively superficial areas of brain, but tDCS can stimulate unto deep areas of brain while being non-invasive, which is widely recognized by neuromedicine community as brain stimulation device. ${ }^{19}$

tDCS is convenient and induces and maintains selective functional alternation in brain cortex. With its mechanism of modulating excitatory with electromagnetic field bypassing scalp and cranium, it is used in various fields of research and exercise. ${ }^{20}$ Sawaki ${ }^{21}$ has stated that various stimulation of peripheral nervous system and central nervous system improves brain neuro-plasticity and further studies are required on various subjects. Recently, clinical experiment and animal experiment are being conducted in regard totDCS and more studies are proving its relation to brain motor region in general population. ${ }^{22}$ Boggio $^{23}$ have reported that bipolar tDCS on to various brain cortex region in healthy adults has im- proved activation of primary motor cortex, and Ragert ${ }^{24}$ have reported increase in sensitivity after applying tDCS onto primary sensory cortex. Koh-un ${ }^{25}$ applied transcranial direct current stimulation, significant difference in side step test and Burpee test.

Therefore, the purpose of this study is to study influence of tDCS after practicing tDCS to soccer players and measure their lower limb muscle activation and balance ability accordingly and provide fundamental reference for efficient exercise of soccer players.

The anodal electrode was positioned on $\mathrm{CZ}, \mathrm{C} 3, \mathrm{C} 4$ and the cathodal electrodes were placed on C5 and C6, following the international 10-20 EEG System.

\section{METHODS}

\section{Subjects}

This study has selected 30 soccer players of a university located in Mokpo who are without any pathologic condition or surgery history, orthopedic injury or abnormality, neurologic history, problem in hearing or vision, and with voluntary participation. All study subjects have undergone 30 minutes of plyometric training, which is followed by 20 minutes of transcranial direct current stimulation for experiment group and 20 minutes of action observation training for control group. Intervention has been performed 50 minutes each session, 5 sessions a week, for 8 weeks. 15 subjects for experiment group and 15 subjects for control group are randomly selected and their general characteristics is as following in Table 1.

\section{Methods}

Plyometric training was conducted for 30 minutes with some alteration to Myer $^{26}$ method. It started with zigzag running, backward running, vertical jump, agility training, side one-foot jump, and spurt, but its degree of difficulty rose gradually so that it would be similar to a real game. Furthermore, experiment group underwent

Table 1. General characteristics of the subjects

\begin{tabular}{lrrrr}
\hline & Group I $(n=15)$ & Group II $(n=14)$ & & \\
\cline { 2 - 3 } & \multicolumn{3}{c}{$\mathrm{M} \pm \mathrm{SD}$} & $\mathrm{p}$ \\
\hline Age (year) & $19.73 \pm 0.91$ & $19.63 \pm 0.80$ & 0.294 & 0.747 \\
Height $(\mathrm{cm})$ & $175.64 \pm 6.02$ & $177.0 \pm 4.96$ & 0.167 & 0.847 \\
Weight $(\mathrm{kg})$ & $72.39 \pm 4.25$ & $68.44 \pm 4.37$ & 2.085 & 0.142 \\
Career (year) & $7.30 \pm 1.19$ & $7.13 \pm 1.01$ & 1.651 & 0.214 \\
\hline
\end{tabular}

Group I: tDCS group, Group II: action observation training group.

a) Shapiro-wilk. 
trans-cranial direct current stimulation training. tDCS was performed with Halo sport (Halo Neuroscience, USA)(Figure 1). Its electrode is a sponge-like substance that can bend well with size of $6.4 \times 4.4 \mathrm{~cm}$ and stimulate $28 \mathrm{~cm}^{2}$ (Figure 2). The anodal electrode was positioned on $\mathrm{CZ}, \mathrm{C} 3, \mathrm{C} 4$ and the cathodal electrodes were placed on C5 and C6, following the international 10-20 EEG system. ${ }^{27}$ Stimulation was made with $2.0 \mathrm{~mA}$ electric current for 20 minutes in total. Maximum electric density of the transmitted current was $0.071 \mathrm{~mA} / \mathrm{cm}^{2}{ }^{28}$ Halo sport pad was soaked in $0.9 \%$ saline and fixated to a degree that may not make the subjects uncomfortable, then the subjects were instructed to close their eyes in a comfortable posture for the stimulation. Furthermore, control group conducted action observational training. In this study, based on study of Karni ${ }^{29}$ the subjects were to look computer screen placed 30 $\mathrm{cm}$ away from the subjects in a quiet room. The subjects' focus heightened while playing a video. Action observational training is a way of training to improve the actual function through the activation of brain regions when we observe other people's behavior and imitate to practice. Action observational training has neurological base on mirror neuron, and it is a way of learning new motor skill using visual - audio effect by observing other person's performance without actual performing of movement. It was required to the sub-

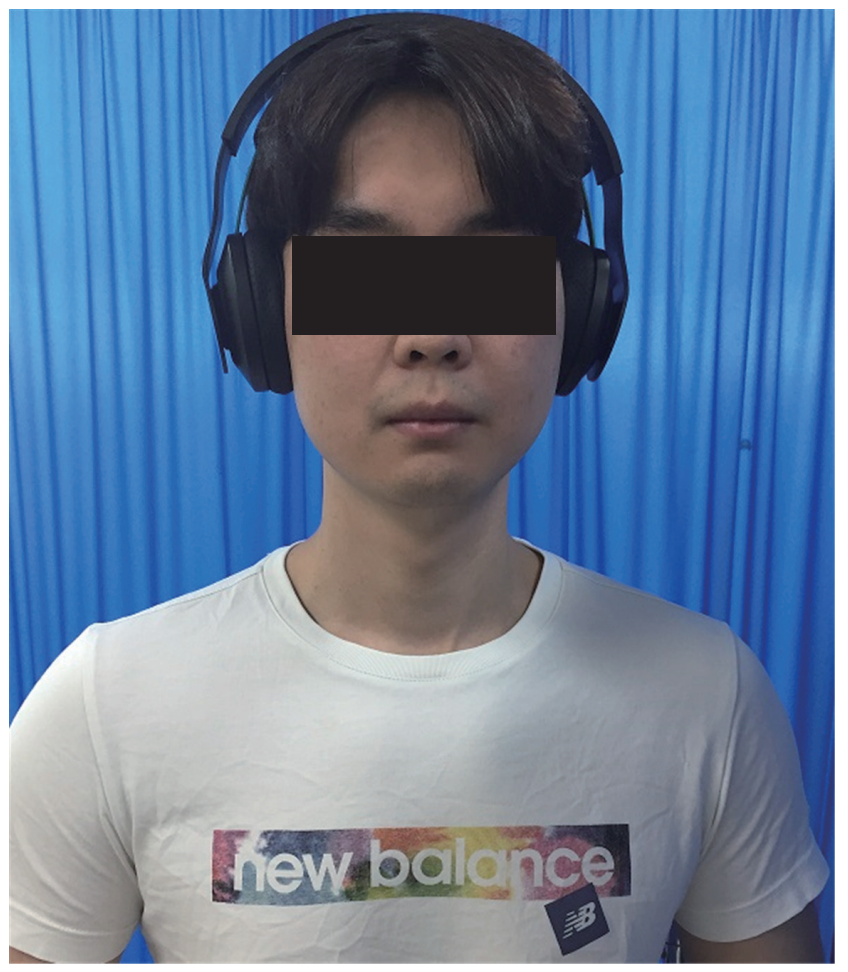

Figure 1. Transcranial direct current stimulation. jects not to follow the contents of the video or move and to focus on the contents of the video. ${ }^{30}$ After observation (5 minutes), the subjects were to reproduce the motion that they saw in video for about 10 minutes. Goal movements per session were limited to 2.

\section{Measurement}

\section{1) Surface electromyogram system}

To measure muscular activation of leg, MP100 surface electromyogram system (Biopac System Inc., USA) was used and digital signals obtained from the device were processed with acknowledge 3.91 program in a personal computer. Bipolar electrodes were attached on the muscles for measurement. Sampling rate of the signals were set at 1,024 Hz and to minimize noise, $60 \mathrm{~Hz}$ notch filter and 30 $500 \mathrm{~Hz}$ band pass filter were employed. Acquired signals were treated with maximum voluntary isometric contraction (MVIC). Hair in area where the electrode would be attached was removed, then skin was scrubbed with fine sandpaper 3-4 times, removing cuticle layer, and cleansed with alcohol to reduce skin resistance to surface electromyogram signals. To standardize action potential of different muscles, muscle activation was measured while making maximum isometric contraction in bare hand MMT test posture. Measurements were taken 3 times for 5 seconds and excluding first and last 1 second, average electromyogram signal for 3 seconds were shown in \%MVIC. Muscles used for the measurement were rectus femoris and biceps femoris. Nienke ${ }^{31}$ argued that among lower limb muscles, rectus femoris has function of important deceleration and extension while biceps femoris assist in flexion and overall action of knee joint, in which collaboration of both muscle groups is to main-

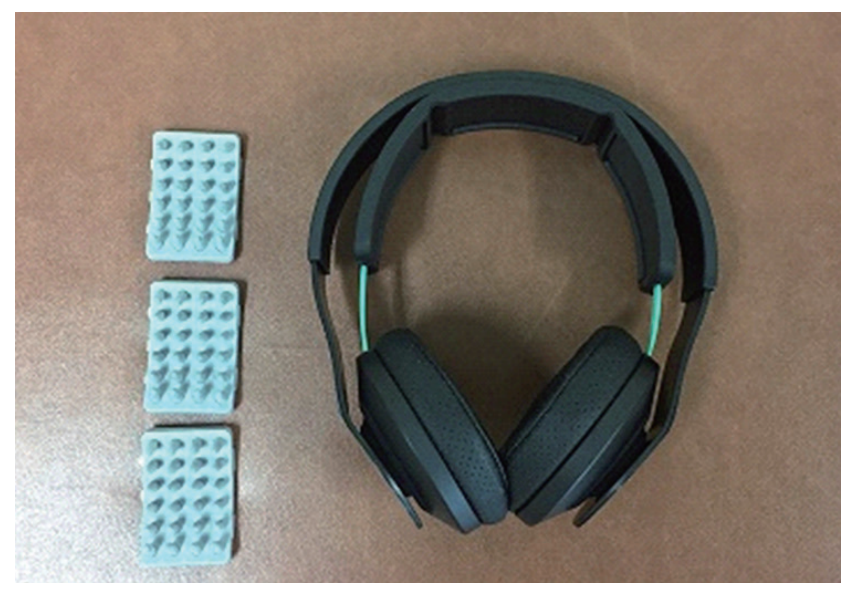

Figure 2. tDCS (Halo sports). 
tain balance of internal and external pressure onto knee joint and reinforcement of both muscle groups would reduce recurrence of injury and improve performance.

\section{2) Balance measurement system}

To analyze balance, Biorescue (RM Ingenierie, France) was used. This device consists of movable square bi-footing force plate and on the plate, a scale is depicted for correct positioning of both feet. To measure balance, the subjects were instructed to look forward for 60 seconds while standing on their both feet, and their surface area of pressure center, whole path length, and limitation of stability were measured. Limited of stability is the maximum limitation to keep stability voluntarily moving in standing posture. Limited of stability is a way to evaluate dynamic balance, and the subject measured the maximum range to keep stability in standing posture. Using the ankle strategy, moving pressure center in 8 directions presented in the monitor, I calculated the area based on the moving distance on center point. All analysis used average value of the results gained from 3 measurements.

\section{Data analysis}

Analysis of the results in this study was processed with Window SPSS 18.0. Shapiro-Wilk was conducted to verify normality of experiment group and control group and analysis of covariance (ANCOVA) was conducted to verify disparity in lower limb muscle activation and balance ability between different intervention groups.
Statistical significance level was set at $\alpha=0.05$.

\section{RESULTS}

\section{Muscle activity}

As for comparison of muscle activation after intervention, rectus femoris of Group I showed improvement from $27.12 \pm 4.02 \%$ to 33.64 $\pm 8.77 \%$ while biceps femoris, $20.33 \pm 3.41 \%$ to $26.41 \pm 4.62 \%$, which showed statistically significant difference from Group II (Table 2).

\section{Balance ability}

As for comparison of balance after intervention, Group I showed change from $26.20 \pm 4.57 \mathrm{~mm}^{2}$ to $23.41 \pm 4.12 \mathrm{~mm}^{2}$ in migration area of surface area, $72.83 \pm 9.49 \mathrm{~cm}$ to $66.34 \pm 8.21 \mathrm{~cm}$ in whole path length, and $904.54 \pm 39.1 \mathrm{~cm}^{2}$ to $967.64 \pm 40.61 \mathrm{~cm}^{2}$ in limited of stability, which show statistically significant difference from Group II (Table 3).

\section{DISCUSSION}

This study has been conducted to study influence of 8 weeks tDCS in muscle activation and balance ability in compare to conventional exercise technique. tDCS has been reported to have significant benefit not only in physical performance, but also in various fields of application, such as conversation skill, memory improvement, chronic pain control, depression, fibromyalgia, and cancer pain. ${ }^{32}$

Table 2. Comparison of muscle activity between groups

(unit: \%MVIC)

\begin{tabular}{|c|c|c|c|c|c|c|}
\hline \multirow{3}{*}{ Muscle } & \multicolumn{2}{|c|}{ Pre-test } & \multicolumn{2}{|c|}{ Post-test } & \multirow{3}{*}{$\mathrm{F}$} & \multirow{3}{*}{$p^{a)}$} \\
\hline & Group I & Group II & Group I & Group II & & \\
\hline & \multicolumn{2}{|c|}{ Mean $\pm S D$} & \multicolumn{2}{|c|}{ Mean $\pm S D$} & & \\
\hline RF & $27.12 \pm 4.02$ & $27.22 \pm 4.21$ & $33.64 \pm 8.77$ & $31.88 \pm 8.41$ & 16.23 & $0.011^{*}$ \\
\hline $\mathrm{BF}$ & $20.33 \pm 3.41$ & $20.68 \pm 3.89$ & $26.41 \pm 4.62$ & $25.41 \pm 4.39$ & 42.43 & $0.013^{*}$ \\
\hline
\end{tabular}

Group I: tDCS group, Group II: action observation training group, RF: rectus femoris, BF: biceps femoris. a) $p=$ ANCOVA; * $p<0.05$.

Table 3. Comparison of balance ability between groups

\begin{tabular}{|c|c|c|c|c|c|c|}
\hline \multirow{3}{*}{ Test } & \multicolumn{2}{|c|}{ Pre-test } & \multicolumn{2}{|c|}{ Post-test } & \multirow{3}{*}{$\mathrm{F}$} & \multirow{3}{*}{$\mathrm{p}^{\mathrm{a})}$} \\
\hline & Group I & Group II & Group I & Group II & & \\
\hline & \multicolumn{2}{|c|}{ Mean $\pm S D$} & \multicolumn{2}{|c|}{ Mean $\pm S D$} & & \\
\hline $\mathrm{SA}\left(\mathrm{mm}^{2}\right)$ & $26.20 \pm 4.57$ & $26.73 \pm 4.98$ & $23.41 \pm 4.12$ & $24.86 \pm 4.49$ & 62.15 & $0.012^{*}$ \\
\hline WPL (cm) & $72.83 \pm 9.49$ & $71.90 \pm 10.74$ & $66.34 \pm 8.21$ & $68.33 \pm 9.02$ & 9.25 & $0.009^{*}$ \\
\hline $\operatorname{LOS}\left(\mathrm{cm}^{2}\right)$ & $904.54 \pm 39.12$ & $910.54 \pm 39.47$ & $967.64 \pm 40.61$ & $955.84 \pm 40.22$ & 21.91 & $0.011^{*}$ \\
\hline
\end{tabular}

Group I: tDCS group, Group Il: action observation training group, SA: surface area, WPL: whole path length, LOS: limited of stability. a) $p=$ ANCOVA; ${ }^{*} p<0.05$. 
tDCS can modulate activation of motor cortex in human brain and is one of the safest, most convenient, and cheapest methods among clinically available methods. However, since there is no exact protocol on indication of tDCS, studies are being conducted on tDCS and tDCS is being reevaluated for its modulation in human brain cortex function with brain stimulation. ${ }^{33}$ It may increase exercise outcome during brain stimulation and such technique allows painless brain stimulation along with various functional alteration depending on intensity, duration, and location of the stimulation. ${ }^{34}$ Though it varies depending on intensity and duration of electric current, generally, single treatment of tDCS for 10-20 minutes can have effect for 30 minutes to 1 hour. It is reported that the effect lasts for 1-2 weeks after repetitive application more than 1 week. ${ }^{35}$

In this study, both group with tDCS and group with action observational training showed significant difference in muscular activation of rectus femoris and biceps femoris. However, tDCS group showed more improvement. As for balance ability, though both tDCS group and action observation training group showed significant difference after intervention, but group with tDCS showed more improvement.

Study by Nitsche ${ }^{19}$ argued activation of overall motor cortex area is further increased after tDCS in normal person for more efficient and selective stimulation of cortex region and such increase can be also occur not only in arms, but also legs. According to Nitche, ${ }^{33}$ alteration in brain cortex activation from briefly applied tDCS lasts for at least 1 hour in brain cortex. Tanaka ${ }^{36}$ confirmed increase in muscular strength of rectus femoris in stroke patients after tDCS, with the effect lasting about 30 minutes. In this study, identical result of enhanced lower limb muscle activation was found in group with tDCS. For more efficient treatment of exercise execution in patients with CNS damage, Hummel \& Cohen $^{37}$ emphasized efficiency in selective stimulus of damaged motor cortex during rehabilitation training. Though preceding study selected patients with CNS damage whereas current study selected soccer players as subjects, Beck ${ }^{16}$ reported that tDCS is beneficial in not only CNS patients and athletes, but also children, adult, and elderly. This is considered to be because of heightened susceptibility to signal from muscle from alteration in excitatory of brain cortex, leading to improved muscle activation. In a study by $\mathrm{Chang}^{38}$ where compared tDCS group and sham tDCS group comprising of patients with knee joint osteoarthritis, tDCS group significantly showed more than double improvement in rectus femoris than sham tDCS group. In current study, tDCS group showed significant improvement of rectus femoris in tDCS group. In a study by Koh, ${ }^{25}$ it compared tDCS group and sham tDCS group comprising of 18 healthy adults, and tDCS group showed faster contraction time in rectus femoris, tibialis anterior, semitendinosus, and gastrocnemius and repetition in side step and burpee test. This is considered to be due to direct influence of $\mathrm{tDCS}$ on motor region of brain, giving a significant effect in lower limb strength. After discharging $1 \mathrm{~mA}$ direct current for $10 \mathrm{~min}$ utes, Lang ${ }^{39}$ reported that motor evoked potential increased 32\% after tDCS than before. Furthermore, Boros ${ }^{40}$ measured motor evoke potential in primary sensorimotor area after applying tDCS onto all motor area its amplitude increased significantly after tDCS, showing direct current could facilitate excitatory of near cortex area in hemisphere. In a study of activation and functional enhancement of lower limb in stroke patients, Madhavanet ${ }^{41}$ argued that tDCS on primary motor cortex can increase excitatory of lower limb while Tanaka ${ }^{26}$ confirmed increase in toe strength of 10 healthy adults after tDCS. Application of tDCS has been reported to influence sensory, motor, and cognition, contribute in continuous alteration of postsynaptic neuron and incur long-term memory reinforcement where validity of synapse is augmented for longer period of time from repetitive stimulus.

$\mathrm{Kim}^{42}$ argued that tDCS onto lower limb motor area in brain cortex with lesion increased knee extension strength which was effective in enhancing lower limb strength and reported that increase in muscular strength led to improved balance. In a study of Kamin$\mathrm{ski}^{43}$ where it compared group with tDCS and group with sham tDCS comprised of healthy adult, tDCS group showed significant improvement in dynamic balance. In current study, tDCS group also showed improvement in balance and as the preceding study, increase in lower limb muscular strength was considered to improve balance ability. Monti ${ }^{44}$ reported that tDCS can manipulate excitatory of brain cells and enhance motor learning as well as various functions, such as working memory. Furthermore, many studies reported tDCS can improve physical motor execution. ${ }^{45}$ Yosephi $^{46}$ treated tDCS to elders with high risk of falling off bed and it showed significant increase in stability limitation. In a study by $\mathrm{Kim}^{47}$ where 18 elderlies of 60 or older were compared of tDCS group and sham tDCS group, tDCS group showed significant improvement in dynamic balance. Current study also showed signifi- 
cant improved limited of stability in tDCS group. Reis ${ }^{48}$ argued that tDCS has positive influence in promoting and learning execution of athletic skills. Current study is based on soccer players which is different from preceding studies in that they are based on elderlies, but tDCS has possibility of enhancing balance and is considered to be helpful in improving balance and performance. tDCS alternates activity of brain neuron in a way that is necessary for recovery of motor function, promotes synaptic plasticity, and provides unspecific input for brain cortex motor system. ${ }^{49}$ Functionally, tDCS can enhance lack of exercise training and performance. ${ }^{50}$

Current study studied effect of tDCS by analyzing muscle activation and balance ability in lower limb after conducting 8 weeks of tDCS on college soccer players. Analysis of muscle activity (MVIC) in rectus femoris and biceps femoris showed that tDCS is more effective in increasing muscular activity than action observation training. Also, based on body center migration area, total trajectory length, and stability limitation, tDCS was proven to be more effective in improving balance than action observation training. Therefore, it reached to a conclusion that tDCS improves muscle activation and balance better than action observation training. Further followup study would be necessary to learn if tDCS is relevant to permanent learning of lower limb muscle activation and balance ability.

\section{ACKNOWLEDGEMENTS}

The research was has been conducted by the research grant of Sehan University in 2018.

\section{REFERENCES}

1. Gutierrez GM, Jackson ND, Dorr KA et al. Effect of fatigue on neuromuscular function at the ankle. J Sport Rehabil. 2007;16(4):295-306.

2. Tak HW. The effect plyometric training of twelve week has on physical fitness and IGF-1 of soccer player of middle school. Jeju National University. Dissertation of Master's Degree. 2014.

3. Kim JH, Uhm YH. Effect of ankle stabilization training using biofeedback on balance ability and lower limb muscle activity in football players with functional ankle instability. J Kor Phys Ther. 2016;28(3):189-94.

4. Marcovic G. Does plyometric training improve vertical jump height? A meta-analytical review. Br J Sports Med. 2007;41(6):349-55.

5. Kim JH, Park SK, Kang JI et al. Effects of lumbar stability exercise program on trunk, lower extremity of muscle activity and balance in soccer player. J Kor Soc Phys Ther. 2010;22(5):25-31.

6. Kwak KI, Choi BJ. Effects of dual task training on balance and functional performance in high school soccer players with functional ankle instability. J Kor Phys Ther. 2016;28(4):254-8.

7. Chimera N, Swanik KA, Swanik CB et al. Effect of plyometric training on muscle-activation strategies and performance in female athletes. J Athl Train. 2004;39(1):24-31.

8. da Silva JF, Nakamura FY, Carminatti LJ et al. The effect of two generic aerobic interval training methods on laboratory and field test performance in soccer players. J Strength Cond Res. 2015;29(6):1666-72.

9. Jeong YS, Kim JH. Effects of whole body vibration exercise on lower extremity muscle activity and balance ability in football player with chronic ankle instability. J Kor Phys Ther. 2017;29(6):293-8.

10. Cavanaugh JT, Guskiewicz KM, Giuliani C et al. Detecting altered postural control after cerebral concussion in athletes with normal postural stability. Br J Sports Med. 2005;39(11):805-11.

11. Paillard T, Noe F, Riviere T et al. Postural performance and strategy in the unipedal stance of soccer players at different levels of competition. Br J Sports Med. 2006;41(2):172-6.

12. Pau M, Arippa F, Leban B et al. Relationship between static and dynamic balance abilities in italian professional and youth league soccer players. Phys Ther Sport. 2015;16(3):236-41.

13. Li L, Han J, Waddington G et al. Ankle proprioception and soccer skills in youth recreational players. Med Sci Sports Exerc. 2017;49(5):1029-30.

14. Campos-Vazquez MA, Romero-Boza S, Toscano B et al. Comparison of the effect of repeated-sprint training combined with two different methods of strength training on young soccer players. J Strength Cond Res. 2015;29(3):744-51.

15. Fusco A, Assenza F, Iosa M et al. The ineffective role of cathodal tDCS in enhancing the functional motor out comes in early phase of stroke rehabilitation: an experimental trial. Biomed Res Int. 2014;2014:547290.

16. Roso C, Perlbarg V, Valabregue R et al. Broca’s area damage is necessary but not sufficient to induce after-effects of cathodal tDCS on the unaffected hemisphere in post-stroke aphasia. Brain Stimul. 2014;7(5):62735.

17. Wagner T, Fregni F, Fecteau S et al. Transcranial direct current stimulation: a computer-based human model study. Neuroimage. 2007;35(3):1113-24.

18. Kwon YH, Kim CS, Jang SH. Cortical activation in the human brain induced by transcranial direct current stimulation. J Kor Soc Phys Ther. 2009;21(4):73-9.

19. Nitsche MA, Cohen LG, Wasermann EM et al. Transcranial direct current stimulation: state of the art. Brain Stimul. 2008;1(3):206-23.

20. Kwon YH, Kwon JW, Park SY et al. Cortical activation by transcranial direct current stimulation and functional electrical stimulation in normal subjects: 2 case studies. J Kor Soc Phys Ther. 2011;23(1):77-82.

21. Sawaki L, Wu CW, Kaelin-Lang A et al. Effects of somatosensory stimulation on use-dependent plasticity in chronic stroke. Stroke. 2006;37(1): 246-7.

22. Anke H, Bahram M, Marlen S et al. Errorless and errorful learning modulated by transcranial direct current stimulation. BMC Neurosci. 2011; 12:72.

23. Boggio PS, Zaghi S, Lopes M et al. Modulatory effects of anodal transcranial direct current stimulation on perception and pain thresholds in healthy volunteers. Eur J Neurol. 2008;15:1124-30.

24. Ragert P, Vandermeren Y, Camus M et al. Improvement of spatial tactile acuity by transcranial direct current stimulation. Clin Neurophysiol. 
2008;119:805-11.

25. Koh U, Doo YT, Lee JW. Effects of transcranial direct current stimulation on leg agility of normal adults: a pilot study. J Health Info Stat. 2018; 43(3):231-6.

26. Myer GD, Ford KR, McLean SG et al. The effects of plyometric versus dynamic stabilization and balance training on lower extremity biomechanics. Am J Sports Med. 2006;34(3):445-55.

27. Valer J, Daisuke T, Ippeita D. 10/20, 10/10, and 10/5 systems revisited: their validity as relative head-surface-based positioning systems. Neuroimage. 2007;34:1600 - 11.

28. Tanaka S, Watanabe K. Transcranial direct current stimulation-a new tool for human cognitive neuroscience. Brain Nerve. 2009;61:53-64.

29. Karni A, Meyer G, Jezzard P et al. Functional MRI evidence for adult motor cortex plasticity during motor skill learning. Nature. 1995;377(6545):155-8.

30. Small SL, Buccino G, Solodkin A. The mirror neuron system and treatment of stroke. Dev Psychobiolo. 2010;54(3):293-310.

31. Nienke WW, Michael PM, Timothy EH. Quadriceps and hamstrings strength in athletes. Hamstring and Quadriceps Injuries in Athletes. 2014;15-28.

32. Fecteau S, Pascual-Leone A, Zald DH et al. Activation of prefrontal cortex by transcranialdirect current stimulation reduces appetite for risk during ambiguous decision making. J Neurosci. 2007;27(23):6212-8.

33. Nitsche MA, Schauenburg A, Lang N et al. Facilitation of implicit motor learning by weak transcranial direct current stimulation of the primary motor cortex in the human. J Cogn Neurosci. 2003;15(4):619-26.

34. Boggio PS, Bermpohl F, Vergara AO et al. Go-no-go task performance improvement after anodal transcranial direct current stimulation of the left dorsolateral prefrontal cortex in major depression. J Affect Disord. 2007;101(1-3):91-8.

35. Butts RJ, Kolar MB, Newman-Norlund RD. Enhanced motor skill acquisition in the non-dominant upper extremity using intermittent theta burst stimulation and transcranial direct current stimulation. Front Hum Neurosci. 2014;8:451.

36. Tanaka S, Taskeda K, Otaka Y et al. Single session of transcranial direct current stimulation transiently increases knee extensor force in patient with hemiparetic stroke. Neuro rehabil Neural Repair. 2011;25(6):565-9.

37. Hummel FC, Cohen LG. Non-invasive brain stimulation: a new strategy to improve neuro rehabilitation after stroke? Lancet Neurol. 2006;5(8): 708-12.

38. Chang WJ, Bennell KL, Hodges PW et al. Addition of transcranial direct current stimulation to quadriceps strengthening exercise in knee osteoarthritis: a pilot randomised controlled trial. PLoS ONE. 2017;12(6): e0180328.

39. Lang N, Nitsche MA, Paulus W et al. Effects of transcranial direct current stimulation over the human motor cortex on corticospinal and transcallosal excitability. Exp Brain Res. 2004;156(4):439-43.

40. Boros K, Poreisz C, Munchau A et al. Premotor transcranial direct current stimulation (tDCS) affects primary motor excitability in humans. Eur J Neurosci. 2008;27(5):1292-300.

41. Madhavan S, Rogers LM, Stinear JW. A paradox: after stroke, the nonlesioned lower limb motor cortex may be maladaptive. Eur J Neurosci. 2010;32(6):1032-9.

42. Kim YU. The effect of tDCS on the postural stability and strength of lower extremity in hemiplegic stroke patients. Chungnam National University. Dissertation of Master's Degree. 2013.

43. Kaminski E, Steele CJ, Hoff M et al. Transcranial direct current stimulation over primary motor cortex leg area promotes dynamic balance task performance. Clin Neurophysiol. 2016;127(6):2455-62.

44. Monti A, Cogiamanian F, Marceglia S et al. Improved naming after transcranial direct current stimulation in aphasia. J Neurol Neurosurg Psychiatry. 2008;79(4):451-3.

45. Cogiamanian F, Marceglia S, Ardolino G et al. Improved isometric force endurance after transcranial direct current stimulation over the human motor cortical areas. Eur J Neurosci. 2007;26(1):242-9.

46. Yosephi MH, Ehsani F, Zoghi M et al. Multi-session anodal tDCS enhances the effects of postural training on balance and postural stability in older adults with high fall risk: primary motor cortex versus cerebellar stimulation. Brain Stimul. 2018;11(6):1239-50.

47. Kim SJ. Whole body dynamic balance for older adults according to transcranial direct current stimulation. Korean Journal of Adapted Physical Activity. 2017;25(2):119-30.

48. Reis J, Schambra HM, Cohen LG et al. Noninvasive cortical stimulation enhances motor skill acquisition over multiple days through an effect on consolidation. Proc Natl Acad Sci U S A. 2009;106(5):1590-95.

49. Zimerman M, Nitsch M, Giraux P et al. Neuroenhancement of the aging brain: restoring skill acquisition in old subjects. Ann Neurol. 2013;73(1): 10-5.

50. Antal A, Varga ET, Nitsche MA et al. Direct current stimulation over MT+/V5 modulates motion after effect in humans. Neuroreport. 2004; 15(16):2491-4. 